
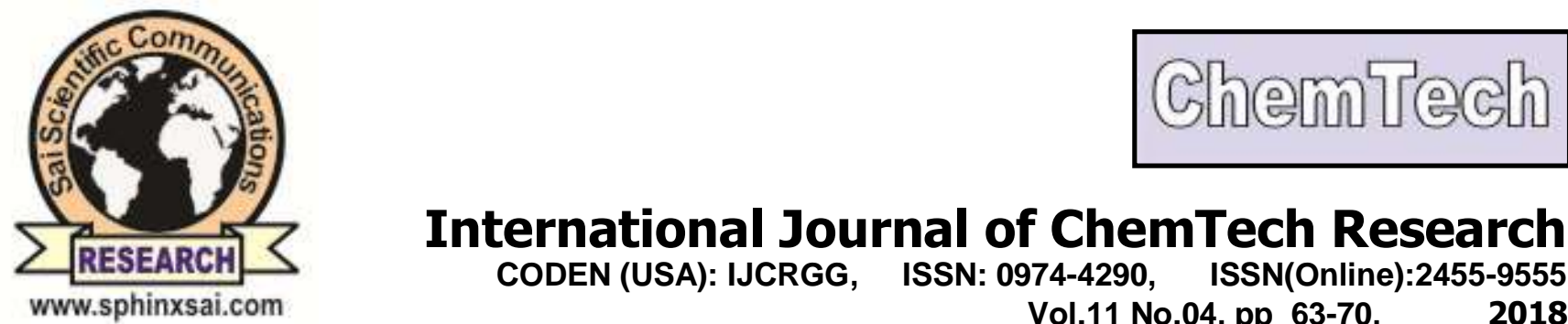

International Journal of ChemTech Research CODEN (USA): IJCRGG, ISSN: 0974-4290, ISSN(Online):2455-9555

\title{
Automatic Railway Gate Control System Using 8051micro Controller
}

\author{
C.R.Balamurugan*, P.Vijayshankarganth, R.Alagarraja, \\ V.E.Subramanian, R.Ragupathy
}

Department of EEE/Karpagam College of Engineering/Coimbatore, India

\begin{abstract}
The objective of this paper is to provide an automatic railway gate at a level crossing replacing the gates operated by the gatekeeper. It deals with two things. Firstly, it deals with the reduction of time for which the gate is being kept closed, and secondly, to provide safety to the road users by reducing the accidents. By the presently existing system once the train leaves the station, the stationmaster informs the gatekeeper about the arrival of the train through the telephone. Once the gatekeeper receives the information, the closes the gate depending on the timing at which the train arrives. Hence, if the train is late due to certain reasons, then gate remain closed for a long time causing traffic near the gates. By employing the automatic railway gate control at the level crossing the arrival of the train is detected by the sensor placed near to the gate. Hence, the time for which it is closed is less compared to the manually operated gates and also reduces the human labour. This type of gates can be employed in an unmanned level crossing where the chances of accidents are higher and reliable operation is required. Since, the operation is automatic; error due to manual operation is prevented. Automatic railway gate control is highly economical microcontroller based arrangement, designed for use in almost all the unmanned level crossings in the country.

Keywords : Railyway gate; level crossing; unmaned; gate; microcontroller.
\end{abstract}

\section{Introduction}

A level crossing occurs where a railway line is intersected by a road or path on one level, without recourse to a bridge or tunnel. It is a type of at grade intersection. The term also applies when a light rail line with separate right-of-way in reserved track crosses a road in the same fashion. Other names include railway crossing, railroad crossing, road through railroad, train crossing or grade crossing. Early level crossings had a lagman in a nearby booth who would, on the approach of a train, wave a red flag or lantern to stop alralic and clear the tracks. Manual or electrical closable gates that barricaded the roadway were later introduced. The gates were intended to be a complete barrier against instruction of any road traffic onto the railway. In the early days of the railways much road traffic was horse drawn or included livestock. It was thus necessary to provide a real barrier. Thus, crossing gates, when closed to road traffic, crossed the entire width of the road. When opened to allow road users to cross the line, the gates were swung across the width of the railway, preventing any

C.R.Balamurugan et al /International Journal of ChemTech Research, 2018,11(04): 63-70.

DOI : http://dx.doi.org/10.20902/IJCTR.2018.110407 
pedestrians or animals getting onto the line. With the appearance of motor vehicles, this barrier became less effective and the need for a barrier to livestock diminished dramatically. Many countries therefore substituted the gated crossings with weaker but more highly visible barriers and relied upon road users following the associated warning signals to stop.

Present work is designed using 8051 microcontroller to avoid railway accidents happening at unattended railway gates, if implemented in spirit. This paper utilizes two powerful IR transmitters and two receivers; one pair of transmitter and receiver is fixed at upside (from where the train comes) at a level higher than a human being in exact alignment and similarly the other pair is fixed at down side of the train direction. Sensor activation time is so adjusted by calculating the time taken at a certain speed to cross at least one compartment of standard minimum size of the Indian railway.

We have Automatic Railway Gate Control System considered 5 seconds for this paper. Sensors are fixed at $1 \mathrm{~km}$ on both sides of the gate. We call the sensor along the train direction as 'foreside sensor' and the other as 'after side sensor'. When foreside receiver gets activated, the gate motor is turned on in one direction and the gate is closed and stays closed until the train crosses the gate and reaches aft side sensors. When aft side receiver gets activated motor turns in opposite direction and gate opens and motor stops. Buzzer will immediately sound at the fore side receiver activation and gate will close after 5 seconds, so giving time to drivers to clear gate area in order to avoid trapping between the gates and stop sound after the train has crossed.

\section{Block Diagram}

This circuit is a small $5 \mathrm{~V}$ power supply, which is useful when experimenting with digital electronics, and easy to build. Small inexpensive wall transformers with variable output voltage are available them any electronics and supermarket. Those transformers are easily available, but usually their voltage regulation is very poor, which makes then not very usable for digital circuit experimenter unless a better regulation can be achieved in some way. The following circuit is the answer to the problem. This circuit can give $+5 \mathrm{~V}$ output at about $150 \mathrm{~mA}$ current, but it can be increased to I A when good cooling is added to 7805 regulator chip. The circuit has over overload and terminal protection.

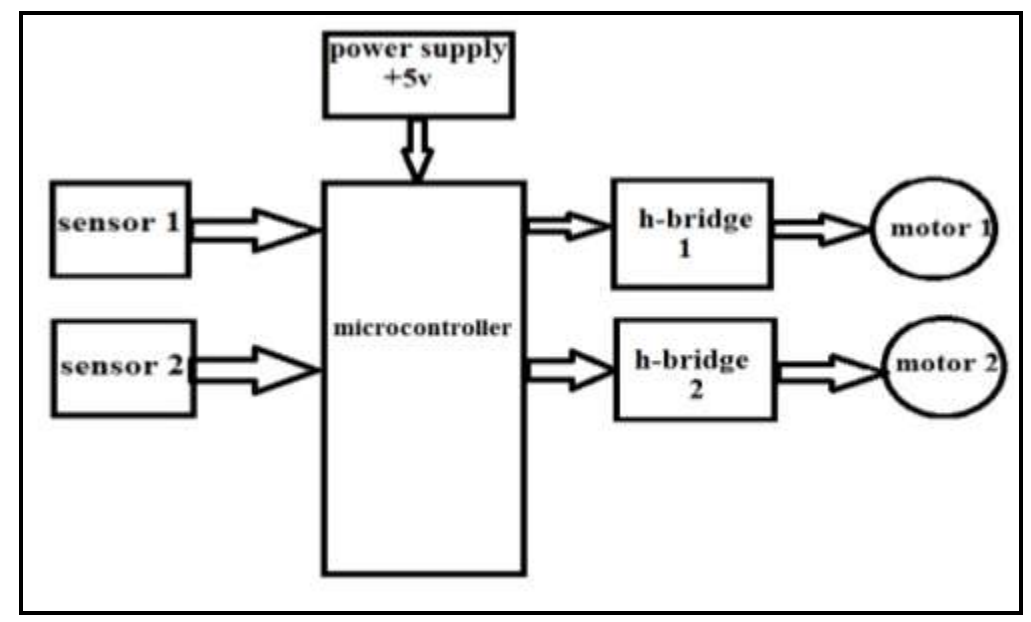

Fig. 1. Block Diagram

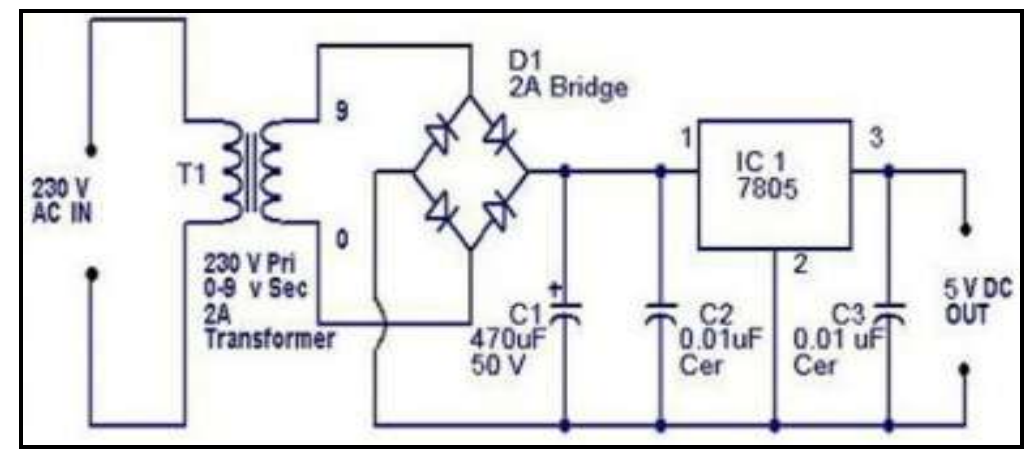

Fig. 2. Power Supply 


\section{a) IR Module}

An infrared sensor is an electronic device that emits in order to sense some aspects of the surroundings. An IR sensor can measure the heat of an object as well as detects the motion. These types of sensors measures only infrared radiation, rather than emitting it that is called as a passive IR sensor Usually in the infrared spectrum, all the objects radiate some form of thermal radiations.

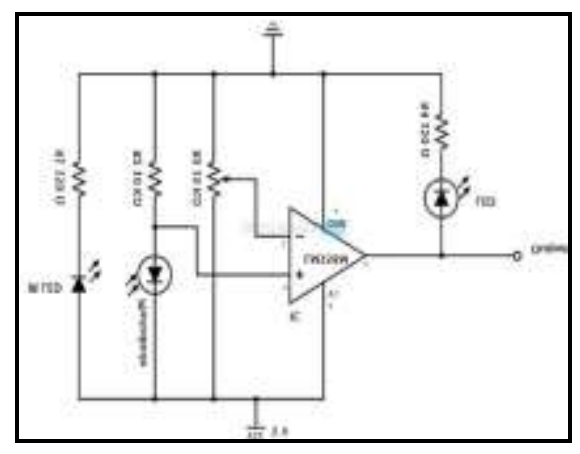

Fig. 3. IR Module

These types of radiations are invisible to our eyes that can be detected by an infrared sensor. The emitter is simply an IR LED (Light Emitting Diode) and the detector is simply an IR photodiode which is sensitive to IR light of the same wavelength as that emitted by the IR LED. When IR light falls on the photodiode, the resistances and these output voltages, change in proportion to the magnitude of the IR light received.

\section{b) H-Bridge Circuit}

The basic operating mode of an H-bridge is fairly simple if Q1 and Q4 are turned on the left lead of the motor will be connected to the power supply, while the right lead is connected to ground Current starts blowing through the motor which energizes the motor in (let's say) the forward direction and the motor shaft starts spinning.
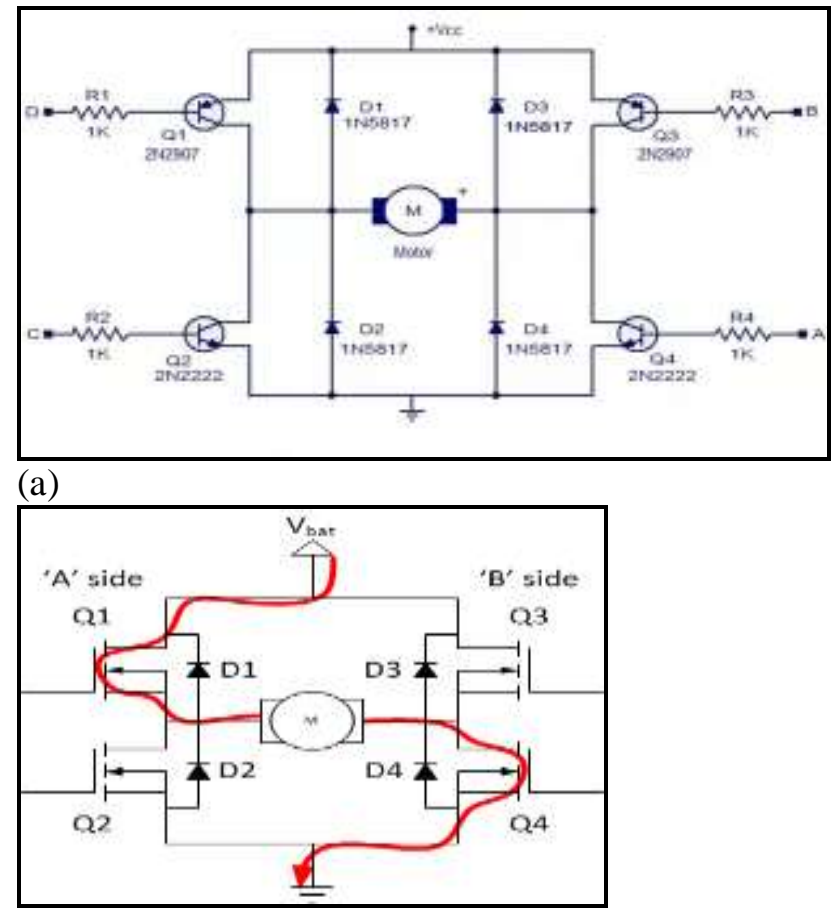

(b) 


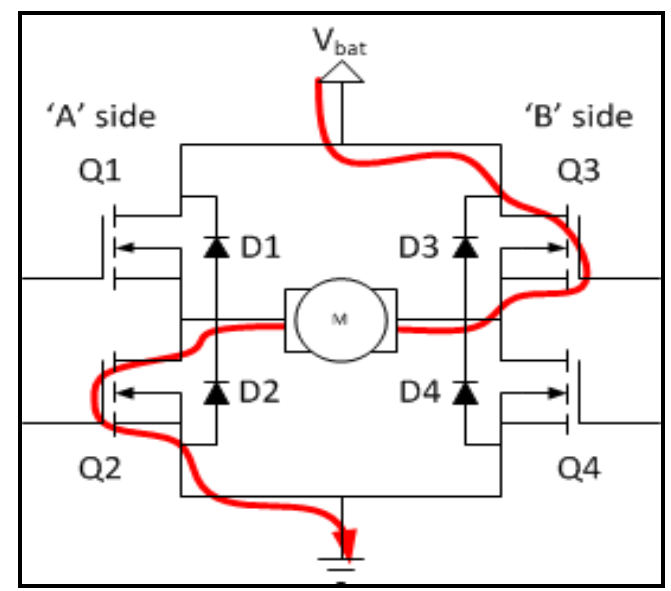

(c)

Fig. 4. H- Bridge Circuit

If Q2 and Q3 are turned on, the reverse will happen, the motor gets energized in the reverse direction, and the shaft will start spinning backwards.

\section{c) Buzzer}

A buzzer or beeper is an audio signaling device, which may be mechanical, electromechanical, or piezoelectric. Typical uses of buzzers and beepers include alarm devices, timers, and confirmation of user input such as a mouse click or keystroke.

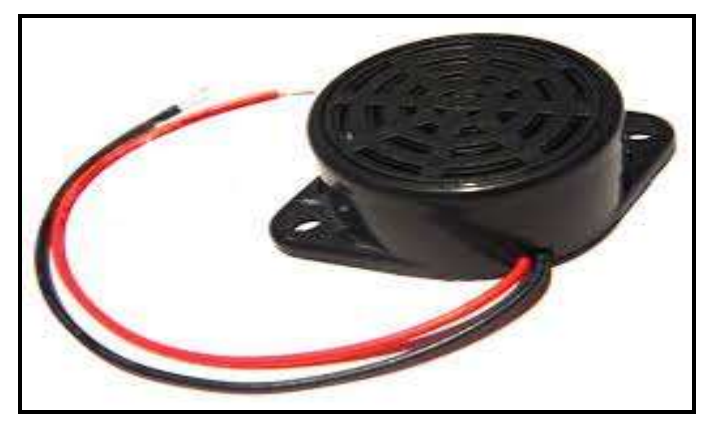

Fig. 5. Buzzer

\section{d) DC Motor}

10RPM, 5V, DC geared motors for robotics applications. Very easy to use and available in standard size. Nut and threads on shaft to easily connect and internal threaded shaft for easily connecting it to wheel.

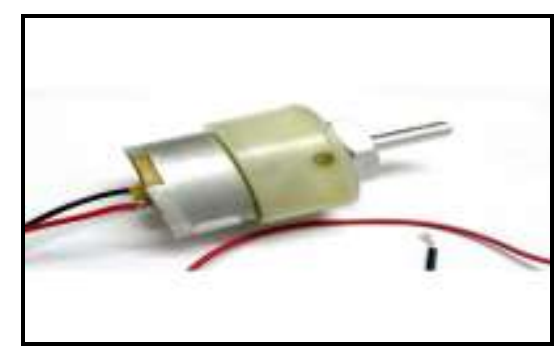

Fig. 6. DC Motor

\section{Circuit Diagram And Componenets}

A micro controller is an integrated circuit or a chip with a processor and other support devices like program memory, data memory, I/O ports, serial communication interface etc integrated together. Unlike a 
microprocessor (ex:Intel 8085), a microcontroller does not require any extremely interfacing of support devices. Intel s051 is the most popular microcontroller ever produced in the world market.

Microcontrollers will combine other devices such as

- A timer module to allow the microcontroller to perform tasks for certain time periods

- A serial I O port to allow data to flow between the controller and other devices such as PIC or another microcontroller

- $\quad$ An ADC to allow the microcontroller to accept analog input data for processing

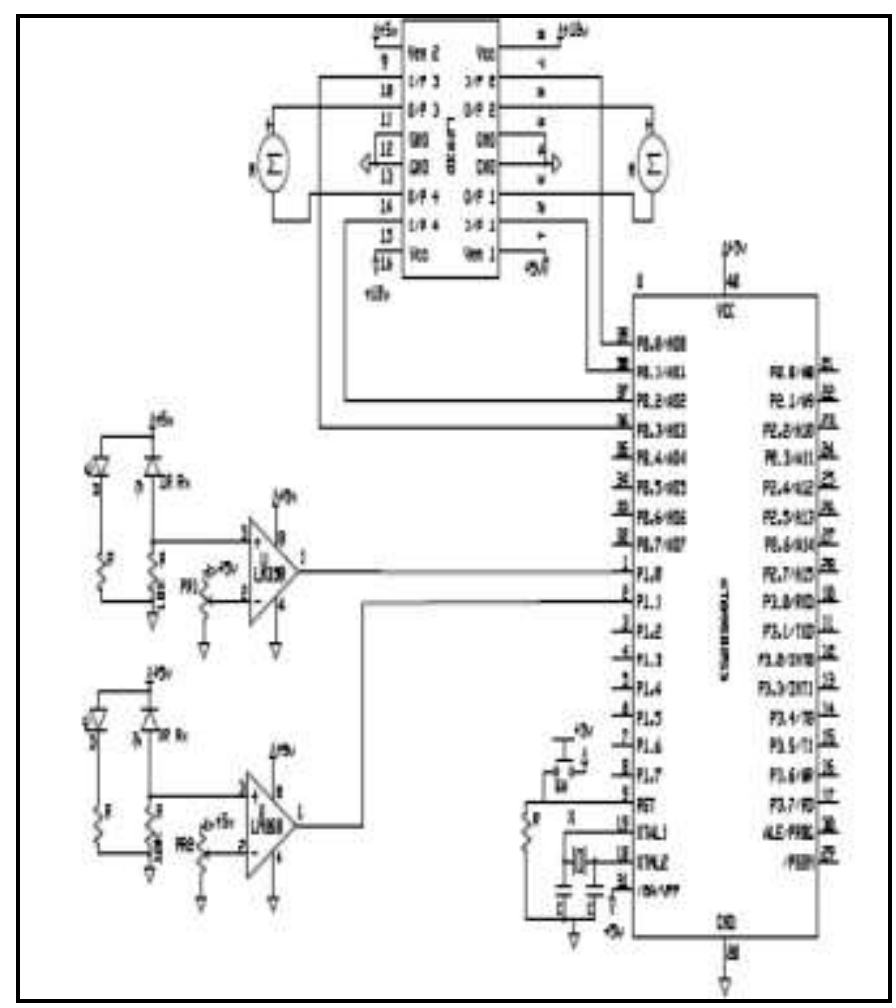

Fig. 7. Circuit Diagram

\section{Complier And Source Code Used}

Keil Software, world's leading developer of Embedded Systems Software, makes ANSI C compilers, macro assemblers, real-time kernels, debuggers, linkers, library managers, simulators, integrated environments, and evaluation boards for the 8051, 251, ARM7, and C16x/ST10 microcontroller families. Keil Software implemented the first $\mathrm{C}$ compiler designed from the ground-up specifically for the 8051 microcontroller.

\#include<reg52.h>

\#include<studio.h>

sfr $\mathrm{p} 0=0 \mathrm{x} 80$;

sfr $\mathrm{p} 1=0 \mathrm{x} 90$

/* LCD interface */

sfr $\mathrm{p} 2=0 \mathrm{xa} 0$

/* for general control */

sfr $\mathrm{p} 3=0 \mathrm{xb} 0$

/* for drive motor drive */

void forward(void);

/* sensor interface additional controls */

void backward(void);

/*carriage motor*/

void delay(ms);

unsigned long $\mathrm{i}, \mathrm{j}$;

sbit drive $1=\mathrm{p} 3^{\wedge} 1$;

sbit drive $2=\mathrm{p} 3^{\wedge} 2$;

sbit led $1=\mathrm{p} 2^{\wedge} 0$;

sbit led2 $=\mathrm{p} 2 \wedge 1$;

void delay(intms) \{

// drive1 buffer output

// drive2 buffer output

//entry sensor input

// exit sensor input 


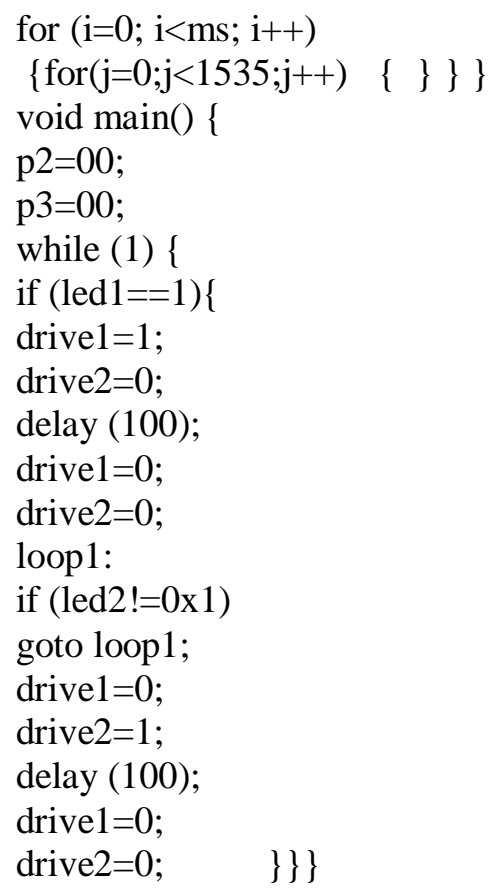

\section{Hardware Circuit}

Railways being the cheapest mode of transportation are preferred over all the other means when we go through the daily news we come across many railway accidents occurring at unmanned railway crossings. This is mainly due to the carelessness in manual operations or lack of workers. We, in this paper have come up with a solution for the same. Using simple electronic components we have tried to automate the control of railway gates. As a train approaches the railway crossing from either side, the sensors placed at a certain distance from the gate detects the approaching train and accordingly controls the operation of the gate. Also an indicator light has been provided to alert the motorists about the approaching train. The two IR sensors are placed at left and right side of the railway gate. The distance between the two IR sensors is dependent on the length of the train. In general we have to consider the longest train in that route. Now we'll see how this circuit actually works in real time. we can see the real time representation of this paper. If the sensor I detects the arrival of the train, microcontroller starts the motor with the help of motor driver in order to close the gate. The gate remains closed as the train passes the crossing. When the train crosses the gate and reaches second sensor. It detects the train and the microcontroller will open the gate.

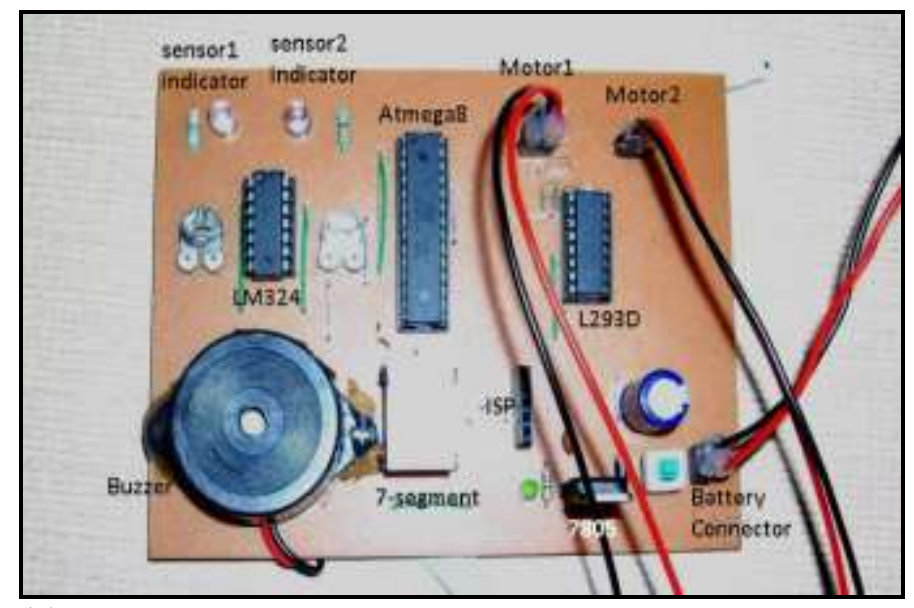

(a) 


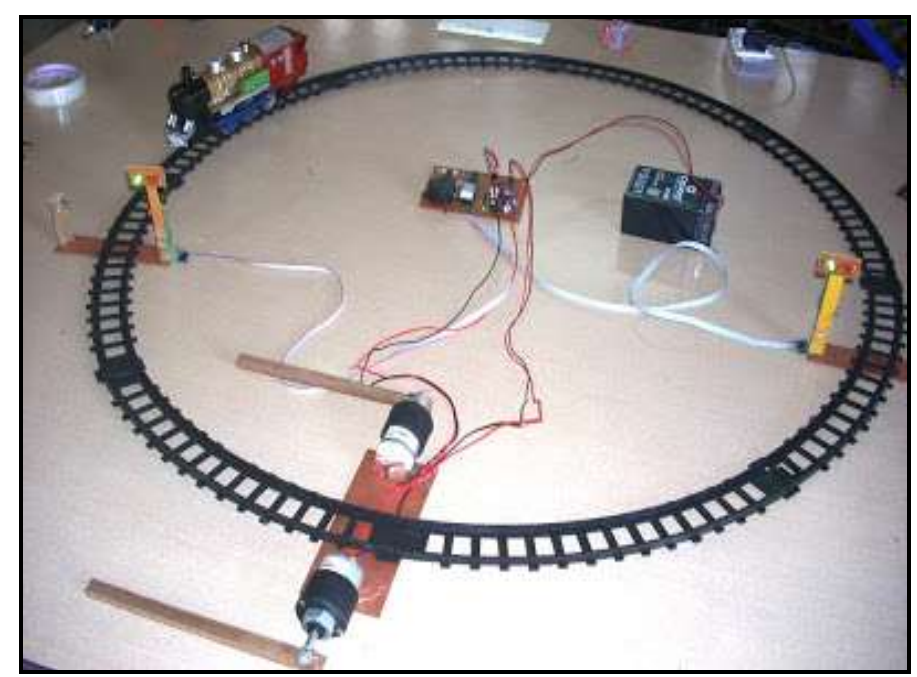

(b)

\section{Fig. 8. Hardware Module}

\section{Conculsions}

The proposed work has many major advantages it will reduce the accidents occurring at the railway level crossing, it will increase the accuracy and reduce errors occurring due to manual operations. It will reduce the collision of train and will also manage the route of a particular train to avoid any delay in reaching its destination. Train will always be on time at the station no delay will be caused which occurs in manual operation. Security can be implemented by placing tracker in the train in order to monitor the location of the train in case of any issue. Solar panels can be used to generate power for the system there by increasing the efficiency of the system. As the system is completely automated, it avoids manual errors and thus provides ultimate safety to road users. By this mechanism, presence of a gatekeeper is not necessary and automatic operation of the gate through the motor action is achieved. Microcontroller 8051 performs the complete operation i.e., sensing, gate closing and opening operation is done by software coding written for the controller. The mechanism works on a simple principle and there is not much of complexity needed in the circuit. Thus the automatic railway gate control using 8051 micro controller is work efficiently and its reduces the human work and time. This is the easy to control the railway gate operation and it reduces the occurrence of faults.

\section{Suggestion For Future Work}

- If a vehicle is between the gates, then a GSM module sends the message when an interrupt occurs

- So the train operator Stops the train earlier

- The entire track consists of number of IR modules

- If any obstruction in the track, then the GSM module will send the message to train operator

\section{References}

1. Ajay.V. Deshmukh, Microcontrollers (Theory and Application), Tata McGraw-Hill Publication, 2005.

2. Ahmed Salih Mahdi, Al-Zuhairi, Automatic Railway Gate and Crossing Control based Sensors \&Microcontroller, International Journal of Computer Trends and Technology (IJCTT), 2013, 4; (7). 2013.

3. Banuchandar J, Kaliraj V, Balasubramanian P, Deepa S, Thamilarasi N, Automated Unmanned Railway Level Crossing System, International Journal of Modern Engineering Research (IJMER), 2012, 2 (1); 458-463.

4. Fred Coleman, Young J. Moon, Trapped Vehicle Detection System for Four Quadrant Gates in High Speed Rail Corridors, Transportation Research Record, 2011.

5. Fred Coleman, Young J. Moon, Design of Gate Delay and Gate Interval Time for Four Quadrant Gate System at Railroad-Highway Grade Crossings Transportation Research Record, 2010.

6. Krishna, Shashi Yadav and Nidhi, Automatic Railway Gate Control usingMicrocontroller",Oriental 
Journal Of Computer Science \& Technology, 2013, 6; 4.

7. V.K. Mehta, Principles of Electronics, 11th edition, S Chand and Company, S.R.M University, 2009

8. Muhammad Ali Mazidi, Janice GillispieMazidi, Rolin.D. McKinlay, The 8051 Microcontrollers and Embedded System, 2nd edition Pearson Education, 2008

9. Paul .G. Slade, Electrical Contacts: Principles and Applications, 2nd edition CRC Press Taylor \& Francis group, 2013 